\title{
Diagnostic disparity of previous and revised American Thoracic Society guidelines for idiopathic pulmonary fibrosis
}

\author{
Lee Fidler $M D^{1}$, Shane Shapera $M D^{2,3}$, Shikha Mittoo $M D^{4,5}$, Theodore K Marras $M D^{2,3}$
}

L Fidler, S Shapera, S Mittoo, TK Marras. Diagnostic disparity of previous and revised American Thoracic Society guidelines for idiopathic pulmonary fibrosis. Can Respir J 2015;22(2):86-90.

BACKGROUND: A revised guideline for the diagnosis of idiopathic pulmonary fibrosis (IPF) was formulated by the American Thoracic Society (ATS) in 2011 to improve disease diagnosis and provide a simplified algorithm for clinicians. The impact of these revisions on patient classification, however, remain unclear.

OBJECTIVE: To examine the concordance between diagnostic guidelines to understand how revisions impact patient classification.

METHODS: A cohort of 54 patients with either suspected IPF or a working diagnosis of IPF was evaluated in a retrospective chart review, in which patient data were examined according to previous and revised ATS guidelines. Patient characteristics influencing the fulfillment of diagnostic criteria were compared using one-way ANOVA and $\chi^{2}$ tests.

RESULTS: Revised and previous guideline criteria for IPF were met in 78\% and $83 \%$ of patients, respectively. Revised guidelines modified a classification based on previous guidelines in $28 \%$ of cases. Fifteen percent of patients meeting previous ATS guidelines failed to meet revised criteria due to a lack of honeycombing on high-resolution computed tomography and the absence of a surgical lung biopsy. Patients failing to meet previous and revised diagnostic criteria for IPF were younger.

CONCLUSION: The revised guidelines for the diagnosis of IPF classify a substantial proportion of patients differently than the previous guidelines.

Key Words: Diagnosis; Guidelines; Idiopathic pulmonary fibrosis; Interstitial fibrosis

Tiopathic pulmonary fibrosis (IPF) is the most common form of idiopathic interstitial pneumonia (IIP), a class of diffuse lung diseases that are associated with significant morbidity and mortality (1). Given the median survival of approximately two to three years, timely identification of IPF is important (2). The clinical heterogeneity of various IIPs makes disease diagnosis challenging for physicians. Improper diagnosis may lead to the initiation of combined immunosuppression, which may be beneficial in some IIPs, but potentially harmful to patients with IPF (3). In 2011, revised guidelines were produced by an international panel of experts under the auspices of the American Thoracic Society (ATS) and other respiratory societies, with the objective of providing evidence-based recommendations for the diagnosis and treatment of IPF (4). A diagnostic algorithm was suggested, using clinical presentation, radiology and histopathology emphasizing the importance of multidisciplinary discussion (MDD). These guidelines highlight the ability of highresolution computed tomography (HRCT) to confirm the diagnosis of IPF, even in the absence of a surgical lung biopsy (SLB). This varies from the previous guidelines in 2000, which, in the absence of an SLB, used major and minor clinical criteria to support a diagnosis of IPF (5). Because there are few available data comparing the performance of

\section{La disparité diagnostique entre les lignes directrices passées et révisées de l'American Thoracic Society à l'égard de la fibrose pulmonaire idiopathique}

HISTORIQUE : En 2011, l'American Thoracic Society (ATS) a révisé ses lignes directrices sur le diagnostic de la fibrose pulmonaire idiopathique (FPI) afin d'en améliorer le diagnostic et de simplifier l'algorithme pour les cliniciens. On ne connaît toutefois pas l'effet de ces révisions sur la classification des patients.

OBJECTIF : Examiner la concordance entre les lignes directrices diagnostiques afin de comprendre l'effet des révisions sur la classification des patients. MÉTHODOLOGIE : Les chercheurs ont procédé à l'étude prospective du dossier d'une cohorte de 54 patients ayant soit une FPI présumée, soit un diagnostic temporaire de FPI. Ils ont examiné les données sur les patients d'après les anciennes lignes directrices de l'ATS et leur révision. Ils ont comparé les caractéristiques des patients qui influaient sur le respect des critères diagnostiques au moyen de l'analyse de variance et du test du chi carré.

RÉSULTATS : Dans l'ensemble, $78 \%$ et $83 \%$ des patients respectaient les critères révisés et antérieurs des lignes directrices de FPI, respectivement. Dans $28 \%$ des cas, les lignes directrices révisées modifiaient l'une des classifications des lignes directrices antérieures. Par conséquent, $15 \%$ des patients qui respectaient les anciennes lignes directrices de l'ATS ne respectaient pas les lignes directrices révisées en raison de l'absence de structure en nid d'abeille à la tomodensitométrie à haute résolution et de l'absence de biopsie pulmonaire chirurgicale. Les patients qui ne respectaient ni les critères diagnostiques de FPI antérieurs ni ceux révisés étaient plus jeunes. CONCLUSION : Les lignes directrices révisées sur le diagnostic de la FPI modifient la classification d'une forte proportion de patients par rapport aux lignes directrices antérieures.

previous versus revised diagnostic guidelines for IPF, it is unclear how changes to these guidelines impact those previously diagnosed. We reviewed records of patients with a working or potential diagnosis of IPF in an interstitial lung disease (ILD) clinic, to compare classification according to previous and revised diagnostic criteria.

\section{METHODS}

The present study was performed using data from patients who consented to unspecified future retrospective studies. All patients consented to study involvement over a 14-month period (July 2011 to September 2012) following research ethics board approval. Eligible for inclusion were all patients at the Toronto General Hospital ILD Clinic (Toronto, Ontario) with apparent IIP and either a working diagnosis of IPF (clinician's record of a diagnosis of IPF) or a potential diagnosis of IPF (clinician's record of IPF as a potential cause of ILD). Exclusion criteria included a confirmed diagnosis other than IPF (or clinical impression that IPF was not the diagnosis), a known cause of ILD (connective tissue disease [CTD], inhalation exposures, etc) or a lack of consent to permit data gathering. All of the required information to evaluate ATS diagnostic criteria for IPF was collected. Patient demographics, clinical presentation, imaging reports,

${ }^{1}$ Division of General Internal Medicine, Department of Medicine, University of Toronto; ${ }^{2}$ Division of Respirology, Department of Medicine, University Health Network and Mount Sinai Hospital; ${ }^{3}$ Division of Respirology, Department of Medicine, University of Toronto; ${ }^{4}$ Division of Rheumatology, Department of Medicine, Mount Sinai Hospital and University Health Network; ${ }^{5}$ Division of Rheumatology, Department of Medicine, University of Toronto, Toronto, Ontario

Correspondence: Dr Theodore K Marras, Division of Respirology, Department of Medicine, Toronto Western Hospital, 399 Bathurst Street, 7 East, Toronto, Ontario M5T 2S8. Telephone 416-603-5767, fax 416-603-5375, e-mail ted.marras@uhn.ca 
pathology, physiological testing and blood work were reviewed. Antinuclear antibodies (ANAs), rheumatoid factor (RF) and anticyclic citrullinated peptide (anti-CCP) results were documented. Testing for ANA and anti-CCP was performed using ELISA, while RF titres were quantified using nephelometry. The presence of chronic obstructive pulmonary disease was based on clinical history, smoking history and HRCT evidence of emphysema. Pulmonary hypertension was evaluated using transthoracic echocardiography. Pulmonary function and 6 min walk tests were performed according to ATS standards and recorded in annual intervals. The presence of gastroesophageal reflux disease was determined by a consistent clinical history. Concern for rheumatological disease prompted evaluation by a rheumatologist before disease diagnosis. Imaging and pathology reports were reviewed for a documented diagnosis and specific terminology consistent with revised ATS guidelines for the diagnosis of IPF (4). All pathology was reviewed by experienced pathologists. With few exceptions, HRCT scans were performed at the Toronto General Hospital and reviewed by thoracic radiologists. Cases with ambiguous HRCT reports were each reviewed by three thoracic radiologists for confirmation of radiological criteria. According to previous guidelines, patients were classified as 'IPF' according to either biopsy-based or clinical criteria. For revised guidelines classification, a classification algorithm based on Table 6 and Figure 3 in the revised guidelines were used, with the implicit assumptions that the HRCT and SLB interpretations were correct, and that MDD was needed to clarify a diagnosis only when the algorithm indicated a designation of 'possible IPF' or 'probable IPF' (4). According to revised guidelines, patients were classified as 'IPF' or, in cases of all other designations ('possible IPF', 'probable IPF', 'not IPF'), as 'not IPF' (recognizing that possible and probable cases should be evaluated further in MDDs to achieve a clear decision). A designation of 'possible IPF' was used to describe cases in which the HRCT fulfilled criteria for a 'possible [usual interstitial pneumonia] (UIP)' pattern (according to revised guidelines) and an SLB was not performed. Concordance was defined as a designation of 'IPF' or 'not IPF' (including 'possible IPF' and 'probable IPF') according to both previous and revised guidelines $(4,5)$. For descriptive purposes, characteristics of patient groups according to IPF classification are presented and compared using one-way ANOVA or Mann-Whitney-U tests, as appropriate for continuous variables, and $\chi^{2}$ or Fisher's exact tests, as appropriate, for categorical variables. Statistical analysis was performed using Excel 2007 (Microsoft Corporation, USA).

\section{RESULTS}

Consent for retrospective study had been previously provided by 174 of $208(84 \%)$ patients, 116 of whom were excluded due to the presence of a diagnosis other than IPF. This group included autoimmune/CTD $(n=79)$, idiopathic entities (ie, sarcoidosis, nonspecific interstitial pneumonia) $(n=23)$ and exposure-induced ILD $(n=14)$. An additional three patients were excluded because the clinical impression did not include IPF as a diagnostic possibility; one patient was excluded because no HRCT result was available. Of the 54 patients included, $50(93 \%)$ carried a working diagnosis of IPF and four (7\%) had not received a formal diagnosis despite thorough evaluation, but were believed to potentially have IPF (Figure 1). The majority of patients were male, previous smokers and had gastroesophageal reflux disease (Table 1). An SLB had been performed in 12 of 54 (22\%) patients, including four with definite UIP pattern on HRCT. The four patients with definite UIP pattern on HRCT were all found to have UIP on histological examination. Two patients had ambiguous HRCT reports; therefore, their scans were each re-evaluated by three thoracic radiologists. One patient was determined to have a UIP pattern and the other, a possible UIP pattern. The proportion of patients who underwent an ANA, RF or anti-CCP test were $85 \%, 83 \%$ and $57 \%$, respectively. Positive titres were identified in 14 of 54 (26\%) patients, comprising eight of 54 (15\%) with an elevated RF level (range $12 \mathrm{IU} / \mathrm{L}$ to $36 \mathrm{IU} / \mathrm{L}$ ) and six of $54(11 \%)$ with positive ANA (range 1:40 to 1:320). All patients with positive serologies underwent rheumatology subspecialist assessment; CTD diagnosis was not substantiated in any.

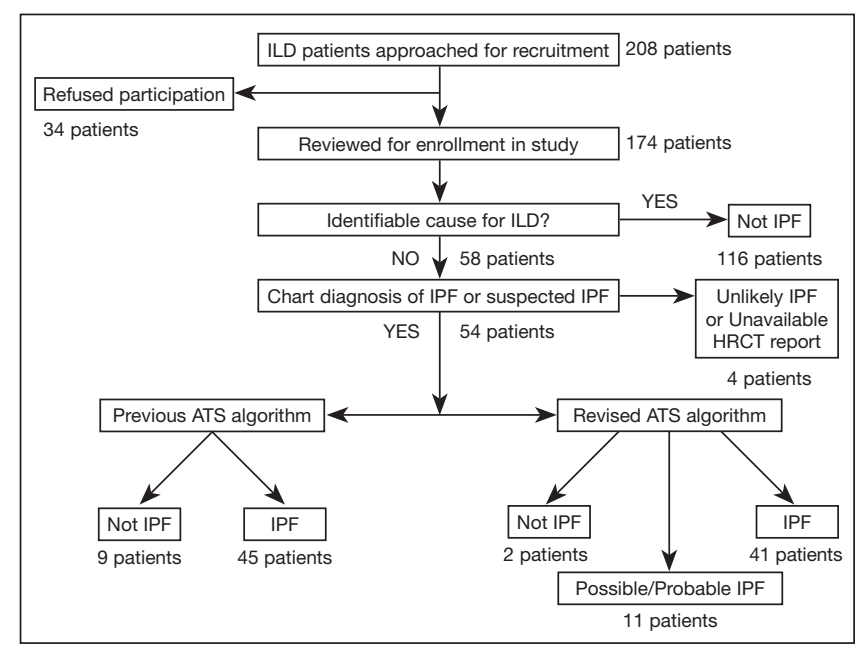

Figure 1) Summary of patient classification according to disease diagnosis and fulfilment of previous and revised American Thoracic Society (ATS) idiopathic pulmonary fibrosis (IPF) criteria. For the present comparison between guidelines, patients classified according to the revised guidelines as possible or probable IPF were categorized as not IPF. HRCT Highresolution computed tomography; ILD Interstitial lung disease

Diagnostic classification was based on the application of the revised and previous guideline algorithms (Appendix 1). Overall, 45 of $54(83 \%)$ and 41 of $54(76 \%)$ patients were diagnosed with IPF according to previous and revised ATS guidelines, respectively, which was not significantly different ( $\mathrm{P}=0.37$ [McNemar test]). Only $37(69 \%)$ patients met the criteria for IPF according to both previous and revised guidelines (Table 2). Eight (15\%) patients met previous ATS criteria, while being classified as possible IPF according to the revised guidelines, mostly based on a lack of honeycombing on HRCT in the absence of an SLB. One subject who underwent SLB met previous guideline criteria; however, under the revised guidelines was classified as possible IPF. Four individuals had IPF according to revised guidelines, but failed to fulfil criteria for IPF in previous guidelines, all due to a lack of major and/or minor criteria in the absence of an SLB. Dyspnea was absent in four patients, while pulmonary function test abnormalities, crackles and symptoms $>3$ months' duration were each not reported in two patients. Five patients did not meet criteria for IPF according to either diagnostic algorithm. Of these five patients, revised guidelines classified three as possible IPF and two as not IPF, while previous guidelines classified all five patients as not IPF. Application of the revised ATS guidelines resulted in a change in diagnosis for 15 (28\%) patients (Figure 2). Revised guidelines introduced the recommendation of MDD, which should ideally be available for all cases, to help decide whether the diagnosis of IPF should be made. The present study used a classification algorithm based on Table 6 and Figure 3 in the revised guidelines, with the implicit assumptions that our HRCT and SLB interpretations were correct, and that MDDs were needed to clarify a diagnosis only when the algorithm indicated a designation of possible or probable IPF (4). According to this construct, the number of patients in this cohort that would require an MDD to further make or refute a diagnosis of IPF according to revised guidelines was $13(24 \%)$. A significant difference in patient age at the time of diagnosis was found between patient groups meeting one, both or neither set of guidelines $(\mathrm{P}=0.006)$ (Table 1$)$.

\section{DISCUSSION}

In our comparison of previous and revised guidelines involving 54 patients with a working or suspected diagnosis of IPF, we found that a similar proportion of patients fulfilled criteria for IPF $83 \%$ and $76 \%$ respectively); however, guideline revision resulted in a change in diagnosis in $28 \%$ of patients. 
TABLE 1

Demographic and clinical characteristics of patients with idiopathic pulmonary fibrosis (IPF) or suspected IPF overall, and according to guidelines classification

\begin{tabular}{|c|c|c|c|c|c|}
\hline \multirow[b]{2}{*}{ Patient characteristic } & \multirow[b]{2}{*}{$\begin{array}{l}\text { All patients } \\
\quad(n=54)\end{array}$} & \multicolumn{3}{|c|}{$\begin{array}{l}\text { Classification according to previous and } \\
\text { revised diagnostic criteria }\end{array}$} & \multirow[b]{2}{*}{$P^{*}$} \\
\hline & & $\begin{array}{l}\text { Concordant IPF } \\
(n=37)\end{array}$ & $\begin{array}{l}\text { Concordant not IPF } \\
\qquad(n=5)\end{array}$ & $\begin{array}{c}\text { Discordant } \\
(n=12)\end{array}$ & \\
\hline Age at disease onset ${ }^{\dagger}$, years, mean & 64 & 64 & 52 & 69 & 0.006 \\
\hline Family history of IPF, $n$ & 1 & 0 & 1 & 0 & 0.33 \\
\hline Gastroesophageal reflux present & $34(63)$ & $25(68)$ & $2(40)$ & $7(58)$ & 0.77 \\
\hline History of chronic obstructive pulmonary disease & $6(11)$ & $4(11)$ & $0(0)$ & $2(17)$ & 0.96 \\
\hline Pulmonary hypertension $\ddagger$ & $16(30)$ & $12(32)$ & $0(0)$ & $4(33)$ & 0.61 \\
\hline Lung cancer & $1(2)$ & $1(3)$ & $0(0)$ & $0(0)$ & 0.33 \\
\hline \multicolumn{6}{|l|}{ Forced vital capacity at presentation, mean } \\
\hline Absolute value, $\mathrm{L}$ & 2.6 & 2.6 & 2.1 & 2.9 & 0.31 \\
\hline Surgical lung biopsy performed & $12(22)$ & $9(24)$ & $2(40)$ & $1(8)$ & 0.66 \\
\hline
\end{tabular}

Data presented as $n(\%)$ unless otherwise indicated. *Continuous variables compared using one-way ANOVA and categorical variables compared using $X^{2}$ or Fisher's exact test (as appropriate); ${ }^{\dagger}$ Disease onset was documented to be the age at the first high-resolution computed tomography scan; ${ }^{\ddagger}$ Echocardiographic data available for 33 of 54 patients overall. 6 MWT 6 min minute walk test; $D L_{C O}$ Diffusion capacity for carbon monoxide

\section{TABLE 2}

Diagnostic classification of 54 patients with clinically likely idiopathic pulmonary fibrosis (IPF) according to previous and revised American Thoracic Society (ATS) guidelines

\begin{tabular}{|c|c|c|c|c|}
\hline \multirow{3}{*}{$\begin{array}{l}\text { Designation } \\
\text { according to } \\
\text { previous ATS } \\
\text { guidelines }\end{array}$} & \multicolumn{3}{|c|}{$\begin{array}{l}\text { Designation according to } \\
\text { revised ATS guidelines }\end{array}$} & \multirow[b]{3}{*}{ Total } \\
\hline & \multirow[b]{2}{*}{ IPF } & Possible IPF & Not IPF & \\
\hline & & \multicolumn{2}{|c|}{ Not IPF } & \\
\hline IPF & 37 (69) & $8(15)$ & $0(0)$ & $45(83)$ \\
\hline Not IPF & $4(7)$ & $3(6)$ & $2(4)$ & $9(17)$ \\
\hline Totals & $41(76)$ & $11(20)$ & $2(4)$ & $54(100)$ \\
\hline
\end{tabular}

Data presented as $n$ (\%)

Focusing on patients with diagnostic discordance may identify potential strengths and limitations of the revised guidelines. Patients classified as IPF by previous guidelines but not by revised guidelines resulted predominantly from the absence of honeycombing on HRCT and a lack of SLB. In total, $15 \%$ of patients fell into this category and were classified as possible IPF. The positive predictive value of HRCT showing subpleural basal reticulation, honeycombing and absence of inconsistent features in the appropriate clinical situation is very high, and the revised guidelines suggest deferral of SLB in such settings $(4,6)$. The shift of patients without honeycombing to a possible IPF category likely improves the specificity of the revised ATS guidelines. This has relevant implications for research and clinical practices. Retrospective research may now be more challenging given that a significant proportion of patients with a chart diagnosis of IPF may only meet possible IPF status on review. However, the included population may be more homogeneous and study conclusions increasingly accurate. The reclassification of patients identified here suggests that previously performed clinical trials may contain a substantial group of subjects who, by current standards, fail to meet revised guidelines for IPF. The theoretical heterogeneity of previously performed study populations may have altered the outcomes of this research. It is reassuring, however, that in a review of diagnoses from a previous IPF trial, among patients with an SLB available, $94 \%$ with a possible UIP

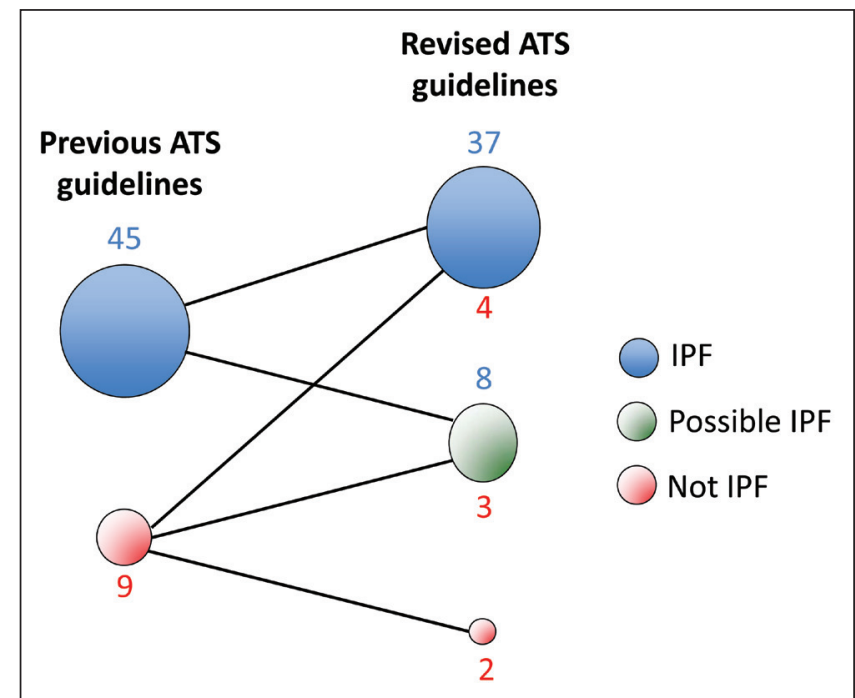

Figure 2) Reclassification of previously diagnosed patients based on application of the revised American Thoracic Society (ATS) guidelines. IPF Idiopathic pulmonary fibrosis

pattern on HRCT had histologically confirmed UIP (7). We suspect that, compared with those meeting previous guidelines, individuals who fulfill revised criteria are more likely to have IPF. This has meaningful clinical implications. The suspected improvement in diagnostic specificity from the revised algorithm may provide physicians with added reassurance when deciding to withhold combination immunosuppressant therapy, which has been shown to be harmful in this population (3).

The substantial number of patients classified as possible IPF based on HRCT results is an important issue. Even after diligent assessment at expert centres, a substantial proportion of patients with ILD will have 'unclassified' ILD (8). There is recognition among experts that categories of possible and probable IPF are problematic as long as specific management guidelines are lacking, and that relevant 


$\begin{array}{ll}\text { TABLE 3 } & \\ \text { American Thoracic Society guideline criteria required for a } \\ \text { diagnosis of idiopathic pulmonary fibrosis (IPF) following } \\ \text { the exclusion of an alternative cause for interstitial lung } \\ \text { disease, } 2000 \text { (4) }\end{array}$

$\overline{\text { FEV }}$ Forced expiratory volume in $1 \mathrm{~s}$; FVC Forced vital capcity; HRCT Highresolution computed tomography; IPF Idiopathic pulmonary fibrosis; PFT Pulmonary function test; UIP Usual interstitial pneumonia; VC Vital capacity

recommendations are needed $(9,10)$. It is useful to consider the positive predictive value of HRCT that lacks honeycombing but otherwise fulfils criteria for a UIP pattern. It has been suggested that even in the absence of honeycombing, as age increases, the probability that IPF is the correct diagnosis can be very high, making age a potentially helpful variable to include in the diagnostic algorithm when honeycombing is not present and an SLB is not available (11). Conversely, high serology titres and younger age are useful predictors in identifying patients at increased risk for developing CTD (12). We found a statistically significant difference in patient age between groups, in which patients failing to fulfill either diagnostic guideline were younger. This is consistent with age being useful as a refuting factor in the diagnosis of IPF. However, patients with discordant diagnoses had the highest mean age, making it difficult to comment on age as a meaningful predictor of IPF based on these results alone. Another factor influencing discordance may have been an increased number of false-positive diagnoses according to previous guidelines, in which the lack of radiographic detail required may have mistakenly elevated the number of patients labelled with IPF.

Patients designated as IPF according to revised guidelines, but not according to previous guidelines, generally had an absence of clinical symptoms fulfilling major and/or minor criteria and lacked an SLB. Radiographic disease can present before clinical manifestations, allowing the diagnosis by revised - but not previous - guidelines. This is a limitation of the previous guidelines, which potentially delays diagnosis until the disease has further progressed.

The rate of SLB in our cohort was low. We suspect this was due to a combination of patient preference and physician hesitancy. Physician hesitancy may result from the perception of surgical complications, as well as a lack of a significant change in therapeutic strategy based on SLB results. In the still-recent era of guidelines recommending a consideration of combined immunosuppressive therapy in IPF, such a position is understandable. With newer data identifying dangers of such treatment in IPF, a definite diagnosis appears to be more desirable (3). The lack of SLB left many patients categorized as possible IPF (before an MDD) according to revised guidelines, while the minor criteria from the former algorithm led to more definitive categorization of IPF versus no IPF. One patient with a pattern inconsistent with UIP on HRCT who underwent SLB was found to have a
TABLE 4

American Thoracic Society guideline criteria required for a diagnosis of idiopathic pulmonary fibrosis (IPF) following the exclusion of an alternative cause for interstitial lung disease, 2011 (5)

\begin{tabular}{lll}
\hline HRCT pattern & $\begin{array}{l}\text { Surgical lung biopsy } \\
\text { (when performed) }\end{array}$ & Diagnosis of IPF \\
\hline UIP & UIP & Yes \\
& Probable UIP & \\
& Possible UIP & \\
& Nonclassifiable fibrosis & \\
& Not UIP & No \\
Possible UIP & UIP & Yes \\
& Probable UIP & \\
& Possible UIP & Probable \\
& Nonclassifiable fibrosis & \\
Inconsistent with UIP & Not UIP & No \\
& UIP & Possible \\
& Possible UIP & No \\
& Probable UIP & \\
& Nonclassifiable fibrosis & \\
& Not UIP & \\
\hline
\end{tabular}

$\overline{\text { HRCT High-resolution computed tomography; UIP Usual interstitial pneumonia }}$

definite UIP pattern on histology. The revised ATS guidelines suggest this patient be labelled as possible IPF, and that MDD be used to clarify whether the diagnosis should be IPF. This situation identifies both the importance of dynamic interaction between experts across several disciplines, and the absence of a widely accessible and simple diagnostic algorithm to classify some patients (Tables 3 and 4).

Limitations of the present study include the lack of certainty in diagnosing IPF, aspects of patient selection and challenges in strictly applying diagnostic guidelines requiring dynamic interaction among experts from multiple disciplines. Currently, the gold standard in diagnosing IPF is an MDD; however, the absence of a confirmatory test for IPF results in unavoidable diagnostic uncertainty. Comparing algorithms may identify strengths and weaknesses of each guideline version without guaranteeing accuracy. The present retrospective study had limitations in confirming aspects of the diagnostic process, specifically, the rigour of MDDs, which are often poorly documented. Accordingly, we selected a pragmatic approach to comparing old and new guidelines through a straightforward review of the clinical data normally available to clinicians. Patients with an obvious cause for their ILD were excluded from our analysis because their diagnoses did not require application of formal diagnostic guidelines. We reasoned that the diagnostic criteria would be most relevant in patients with a significant clinical suspicion for IPF (ie, proven IPF, working or potential diagnosis of IPF), comprising the group in which guidelines would be used. However, our selection criteria may limit the generalizability of our findings. It should also be noted that we did not strictly apply the new guidelines, in that MDD was not applied for all cases, instead opting for a more pragmatic approach. We assumed that our clinical records' data, and HRCT and SLB interpretations were accurate, and that MDD would be needed in cases of possible or probable IPF as outlined in the algorithm from the revised guidelines.

In summary, the application of previous or revised IPF diagnostic guidelines leads to a similar rate of diagnosis, despite considerable discordance among individual cases. In other words, the revised ATS diagnostic criteria led to reclassification of a substantial number of patients in that they have a different probability or certainty of diagnosis of IPF. We believe the revised directives are an improvement from the previous, facilitating earlier disease detection in cases with few symptoms or physiological derangements, and enhancing 
diagnostic specificity. The application of revised ATS guidelines will likely increase the uniformity of research study populations and decrease uncertainty around withholding combined immunosuppression for individuals with IPF.

ACKNOWLEDGEMENTS: LF takes responsibility for the content, data collection and statistical analysis for this article; LF contributed to the study design, collected and analyzed the data, contributed to the interpretation of the data and drafted the manuscript; SS contributed to the study design, interpretation of the data, and critically revised the manuscript for intellectual content; SM contributed to the study design, interpretation of data and critically revised the manuscript for intellectual content; TKM conceived the study, contributed to the study design and interpretation of the data, and critically revised the manuscript for intellectual content. The finalized manuscript was reviewed by all authors before submission.

DISCLOSURES: There are no relevant conflicts of interest to declare for this study. This study has not been presented at any international conferences in abstract or oral form.

FUNDING: This study was funded by the Canadian Pulmonary Fibrosis Foundation.

\section{REFERENCES}

1. du Bois RM. An earlier and more confident diagnosis of idiopathic pulmonary fibrosis. 2012. Eur Respir Rev 2012;21:141-6.

2. Bjoraker JA, Ryu JH, Edwin MK, Tazelaar HD, Schroeder DR, Offord KP. Prognostic significance of histopathologic subsets in idiopathic pulmonary fibrosis. Am J Respir Crit Care Med 1998;157:199-203.

3. Idiopathic Pulmonary Fibrosis Clinical Research Network; Raghu G, Anstrom KJ, King TE Jr, Lasky JA, Martinez FJ. Prednisone, azathioprine, and $\mathrm{N}$-acetylcysteine for pulmonary fibrosis. N Engl J Med 2012;366:1968-77.

4. Raghu G, Collard HR, Egan JJ, et al. An official ATS/ERS/JRS/ ALAT statement: Idiopathic pulmonary fibrosis: Evidence based guidelines for diagnosis and management. Am J Respir Crit Care Med 2011;183:788-824.

5. American Thoracic Society. Idiopathic pulmonary fibrosis: Diagnosis and treatment. International consensus statement. American Thoracic Society (ATS), and the European Respiratory Society (ERS). Am J Respir Crit Care Med 2000;161:646-64.

6. Swensen SJ, Aughenbaugh GL, Myers JL. Diffuse lung disease: Diagnostic accuracy of CT in patients undergoing surgical lung biopsy of the lung. Radiology 1997;205:229-34.

7. Raghu G, Lynch D, Godwin JD, et al. Diagnosis of idiopathic pulmonary fibrosis with high-resolution CT in patients with little or no radiological evidence of honeycombing: Secondary analysis of a randomised, controlled trial. Lancet Respir Med 2014;2:277-84.

8. Ryerson CJ, Urbania TH, Richeldi L, et al. Prevalence and prognosis of unclassifiable interstitial lung disease. Eur Respir J 2013;42:750-7.

9. Wells AU. The revised ATS/ERS/JRS/ALAT diagnostic criteria for idiopathic pulmonary fibrosis (IPF) - practical implications. Respir Res 2013;14(Suppl 1):S2.

10. Travis WD, Costabel U, Hansell DM, et al. An official American Thoracic Society/European Respiratory Society statement: Update on the international multidisciplinary classification of the idiopathic interstitial pneumonias. Am J Resp Crit Care Med 2013;188:733-48.

11. Fell CD, Martinez FJ, Liu LX, et al. Clinical predictors of a diagnosis of idiopathic pulmonary fibrosis. Am J Respir Crit Care Med 2010;181:832-7.

12. Mittoo S, Gelber AC, Christopher-Stine L, Horton MR, Lechtzin N, Danoff SK. Ascertainment of collagen vascular disease in patients presenting with interstitial lung disease. Respir Med 2009;103:1152-8.

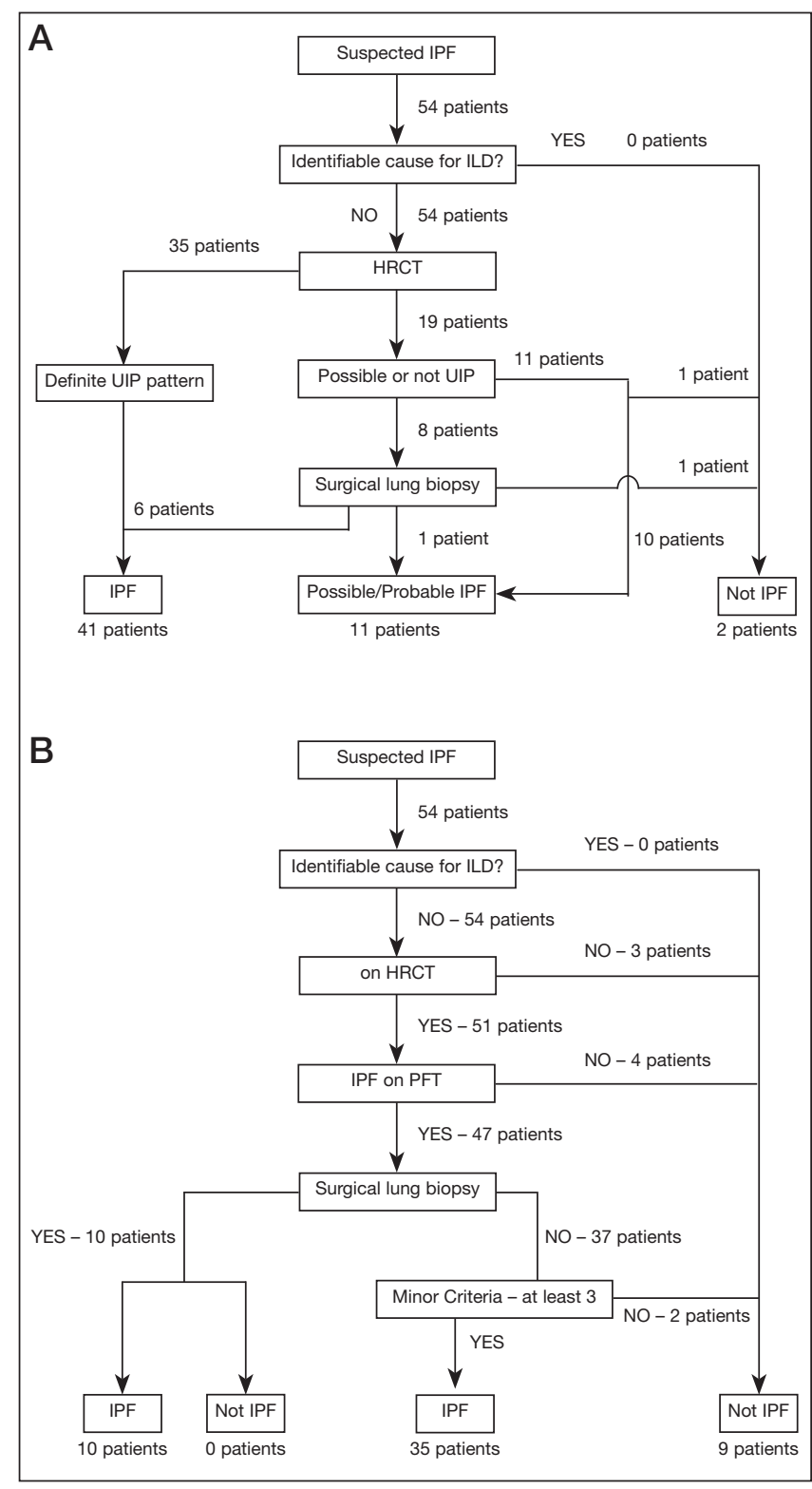

Appendix 1) Distribution of patients according to revised (A) and previous (B) American Thoracic Society (ATS) diagnostic algorithms for idiopathic pulmonary fibrosis (IPF). In panel B, consistent with previous ATS guidelines, IPF on high-resolution computed tomography (HRCT) refers to bibasilar reticular abnormalities with minimal ground-glass opacities. IPF on pulmonary function test (PFT) refers to evidence of restriction (reduced vital capacity, often with an increased forced expiratory volume in 1 s to forced vital capacity ratio) and impaired gas exchange. ILD Interstitial lung disease; UIP Usual interstitial pneumonia 


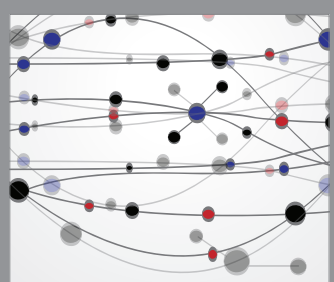

The Scientific World Journal
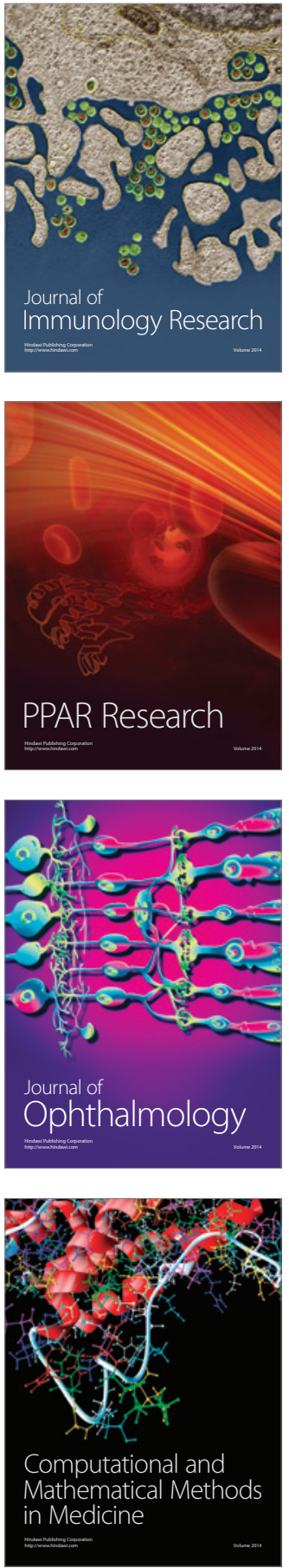

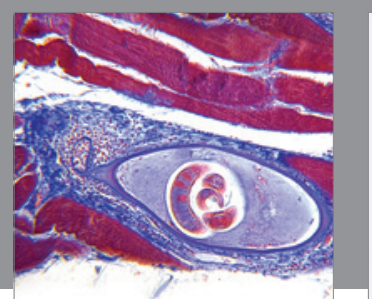

Gastroenterology Research and Practice

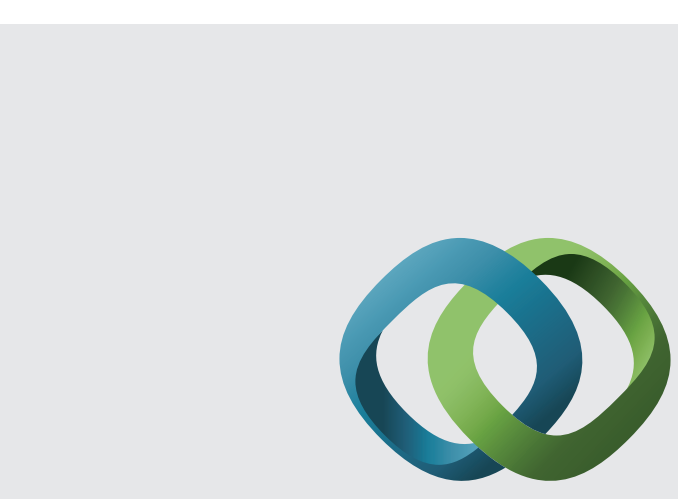

\section{Hindawi}

Submit your manuscripts at

http://www.hindawi.com
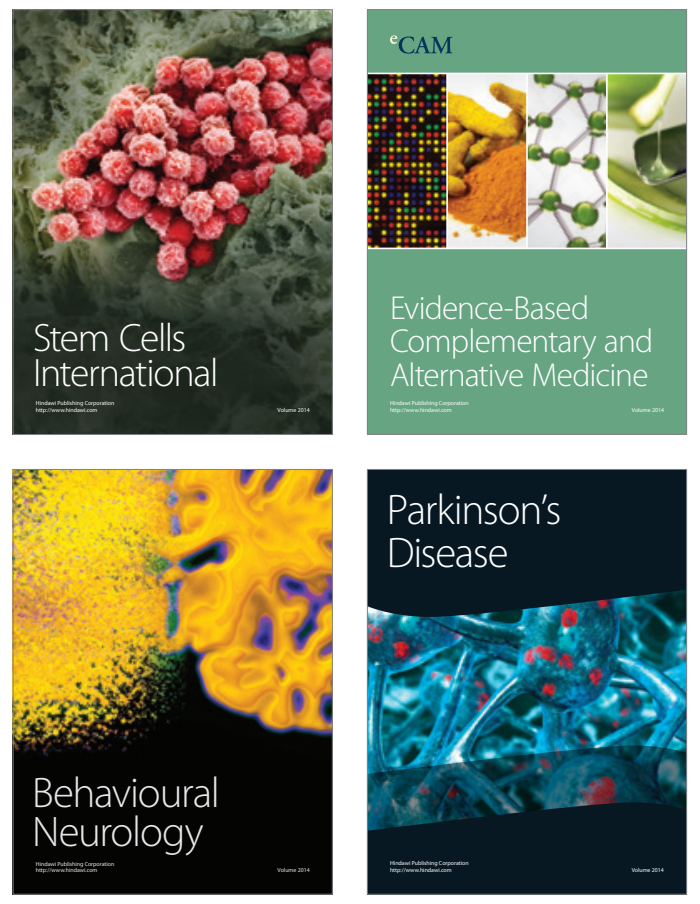
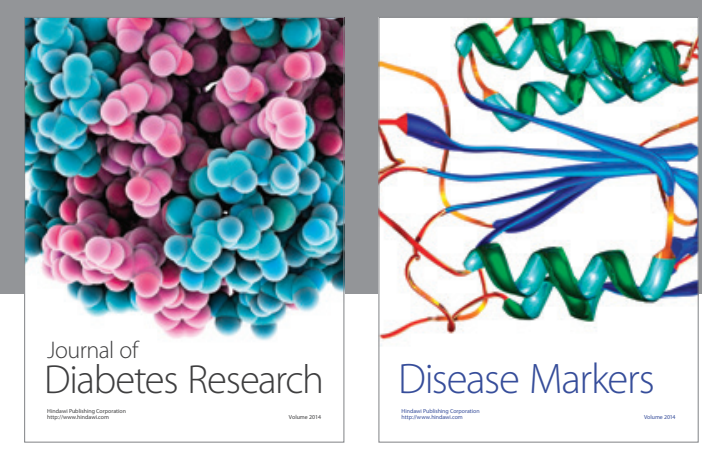

Disease Markers
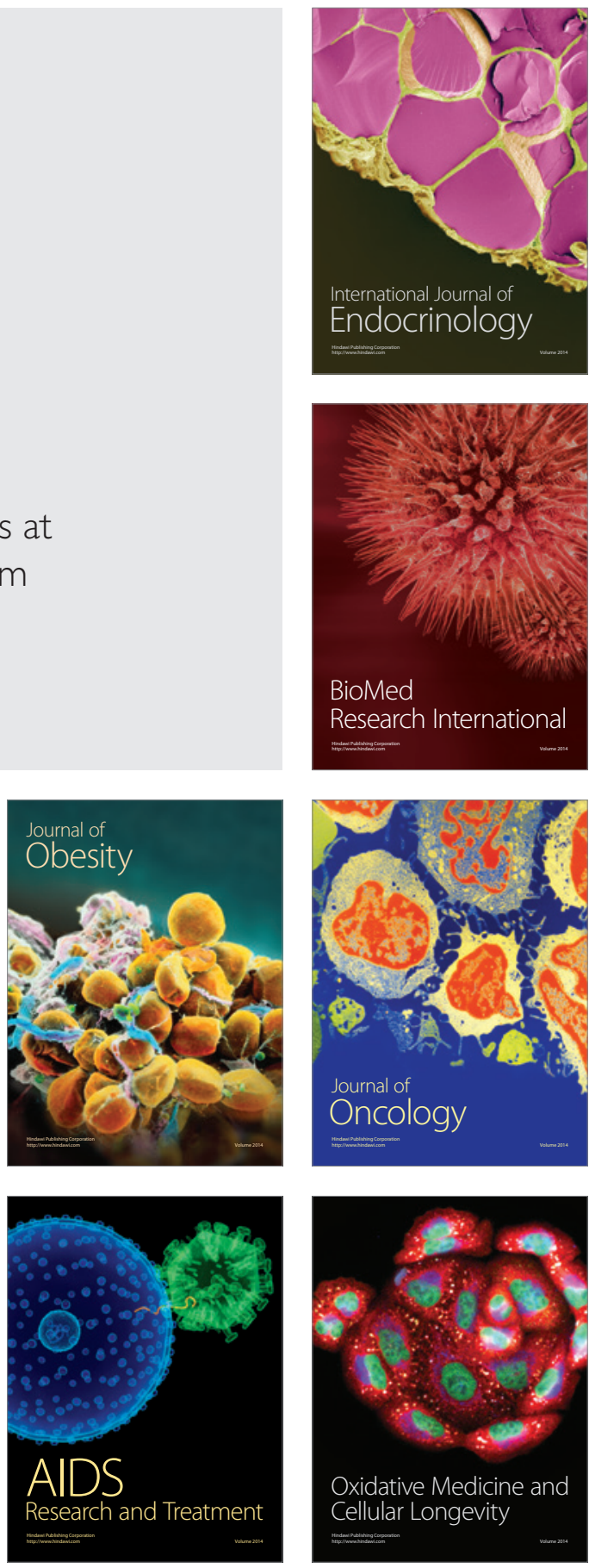